\title{
The price and liquidity impact of china forbidding initial coin offerings on the cryptocurrency market.
}

\author{
Sijia Zhang and Andros Gregoriou* \\ All authors are from Brighton Business School, University of Brighton, Mithras House, \\ Lewes Road, Brighton. BA2 4NT, UK.
}

\begin{abstract}
In this paper we empirically examine the cryptocurrency market reaction to china prohibiting initial coin offerings, on the $4^{\text {th }}$ September 2007 for the 100 largest cryptocurrencies. The announcement has a significant negative but temporary impact on cryptocurrency returns and liquidity.
\end{abstract}

JEL Classification: G14; G15

Key words: Cryptocurrency; Liquidity; Bid-ask spread; Amihud ratio

* Corresponding author a.gregoriou@brighton.ac.uk 


\section{Introduction}

Cryptocurrencies are considerably growing and have attracted significant attention from financial markets since Bitcoin's inception in 2008 by Nakamoto. Cryptocurrency is a complex peer-to-peer electronic cash system, which allows online trading directly between investors without going through a financial institution (Nakamoto, 2008). Cryptocurrencies are extremely popular for investors because of their transparency, trading speed, high liquidity and simplicity in use. The peer-to-peer system and governmental free design provides a new instrument for portfolio allocation to investors (Zargar and Kumar, 2019). In October 2019, there are more than 2000 cryptocurrencies, which contribute to a total market capitalisation of approximately 222 billion USD.

Cryptocurrencies are traded on independently operated trading platforms and are accessible to a global clientele 24 hours a day, 7 days per week. When new information arrives, it is incorporated immediately without waiting for the trading time (Ciaian et al., 2016). Corbet et al. (2018) indicates that cryptocurrencies are uncorrelated with all other major assets, such as gold, oil and equity indices. Dwyer (2015) mentions that the volatility of Bitcoin (the most heavily traded cryptocurrency) is significantly higher than other financial assets.

Considering these unique features of cryptocurrencies mentioned above, we believe that identification of the factors driving the liquidity of cryptocurrencies is an important research question. Liquidity is the ability to convert the cryptocurrency into cash without any difficulty. Brauneis and Mestel (2018) show that the improvement in liquidity leads to the cryptocurrency market becoming more efficient. Kristoufek (2018) states that liquidity levels in cryptocurrencies are relatively low compared to traditional financial markets. 
Our study is the first paper to provide liquidity effects around one of the major negative news announcements in the cryptocurrency markets. The Chinese government banned initial coin offerings (ICOs) on the $4^{\text {th }}$ of September 2017. The Chinese central financial institutions jointly issued the announcement averting ICOs for purposes of investor protection and financial risk prevention (hereafter, ICO rules) ${ }^{1}$. The ICO rules prohibit buying or selling cryptocurrencies, setting prices, or other related agent services. They are also not allowed to convert legal tender into cryptocurrencies or vice versa. China was one of the most important cryptocurrency trading nations in the world. This is reflected by china trading almost $100 \%$ of Bitcoin in 2016 (bitcoinity.org).

The remainder of the paper is organized in the following way. Section 2 describes data and methodology. Section 3 reports the empirical results and finally section 4 concludes.

\section{Data and Methodology}

\subsection{Data}

We collect open, closing prices and market capitalization of cryptocurrencies from coinmarketcap.com. Our data consists of the 100 largest cryptocurrencies with respect to market capitalization. Our quoted price information is from Kaiko.com. In order to capture preannouncement drift, we apply a 5-day pre-event window. As cryptocurrency markets react to news very quickly and are highly volatile, a longer estimation window is at greater risk of anticipating other market forces. Therefore, we use a 20-day post-announcement period. In total, there are 26 event days in our sample period. All the data is specified in US dollars.

\footnotetext{
${ }^{1}$ https://www.nortonrosefulbright.com/en/knowledge/publications/aa676f71/china-issues-announcement-to-banfundraising-through-token-offerings
} 


\subsection{Methodology}

\subsubsection{Abnormal return}

The bad news announcement date (4 September 2017) is the day that the Chinese government issued the policy to ban the ICOs. We examine abnormal returns (ARs) for the announcement day [day 0] and several event windows around the announcement period. Following Zhang et al. (2019), we estimate the cryptocurrency abnormal returns as follows:

$$
\mathrm{AR}_{i, t}=\mathrm{R}_{i, t}-\mathrm{R}_{m, t}
$$

Where $A R_{i, t}$ is the abnormal return earned by cryptocurrency $i$ at time $t$. $R_{i, t}$ is the return on cryptocurrency $i$ at time $t$, and $R_{m, t}$ is the market average return at time $t$. Cumulative abnormal return (CAR) of the event window surrounding the event day can be calculated using the following formula:

$$
\mathrm{CAR}_{i, t(-q,+q)}=\sum_{t=q}^{\mathrm{S}} \mathrm{AR}_{i, t}
$$

Where $q$ represents the different time periods during the event window. The standard t-test is used to examine if $A R_{i, t}$ and $C A R_{i, t}$ are significantly different from zero.

\subsubsection{Bid-ask spread}

We measure the bid-ask spread in the following way:

$$
\mathrm{ES}_{\mathrm{i}, \mathrm{t}}=2\left|\mathrm{P}_{i, t}-\mathrm{Mid}_{i, t}\right|
$$

Where $E S_{i, t}$ is the effective spread of cryptocurrency $i$ at time $t$ respectively. $P_{i, t}$ is the cryptocurrency price of cryptocurrency $i$ at time period $t$ and $\operatorname{Mid}_{i, t}$ is the midpoint between the bid and the ask price of cryptocurrency $i$ at time period $t$.

\subsubsection{Price impact ratio test}

For robustness, we also implement price impact ratios because they capture liquidity from the perspective of financial stability. The Amihud (2002) illiquidity ratio (RtoV) is defined as 
absolute daily return on cryptocurrency $i$, divided by the cryptocurrency's daily monetary volume, average over a trading period:

$$
\mathrm{RtoV}_{\mathrm{i}}=\frac{1}{\mathrm{D}_{i}} \sum_{d=1}^{\mathrm{D}_{i}} \frac{\left|\mathrm{R}_{i, d}\right|}{\mathrm{V}_{i, d}}
$$

Where $\left|R_{i, d}\right|$ and $V_{i, d}$ refers to the absolute return and monetary volume of cryptocurrency $i$ on day $d$ respectively, $D_{i}$ is the number of trading days for cryptocurrency $i$. The RtoV ratio exhibits a significant size bias, since trading volume is positively correlated with market capitalization (Florackis et al., 2011). Given these shortcomings, Florackis et al. (2011) constructs an alternative price impact ratio, defined as the average ratio of daily absolute cryptocurrency return to its turnover ratio (RtoTR ratio):

$$
\mathrm{RtoTR}_{\mathrm{i}}=\frac{1}{\mathrm{D}_{i}} \sum_{d=1}^{\mathrm{D}_{i}} \frac{\left|\mathrm{R}_{i, d}\right|}{\mathrm{TR}_{i, d}}
$$

Where $T R_{i, d}$ refers to the turnover ratio of cryptocurrency $i$ at day $d, D_{i}$ and $R_{i, d}$ are the same as defined in equation (4).

\section{Empirical results}

\subsection{Price effects change in cryptocurrency prices}

From Table 1 we observe a significant decrease in abnormal return associated with the announcement of forbidding ICOs. The negative abnormal returns start from 2 days before the announcement, -0.013 in day -2 and -0.006 in day -1 , with both significant at the $10 \%$ level. The negative abnormal return persists from day -2 until day 4 , with the two most significant negative returns of -4.265 (t-statistic of -11.28) on the event day and -3.057 (t-statistic of -9.99) on the first day after the announcement (day 1) respectively.

The average cumulative abnormal return of -7.327 is observed over the 3 -event day interval [$1,+1]$ with a highly significant t-statistic, at all conventional levels. Event windows $[-2,+2]$, $[-3,+3]$ have CARs of -10.874 and -11.739 percent respectively, with both of them significant 
at the $1 \%$ level. 10 days after the negative news announcement, the abnormal returns are reversed from negative to positive, with values of 0.458 for day 10 and 0.639 for day 20 . This implies that the price effect of China banning ICOs is temporary. Panel B presents the results of the five largest cryptocurrencies (Bitcoin, Ethereum, Ripple, Bitcoin Cash, and EOS). The results are similar with Panel A, indicating that the abnormal returns are significantly influenced by Bitcoin, Ethereum, Bitcoin Cash, EOS and Ripple. Our results also suggest that cryptocurrency markets are efficient, because when information arrives the price is reacting immediately.

\section{[INSERT TABLE 1 HERE]}

\subsection{Changes in illiquidity ratios}

In the first two columns of Table 2, we construct ratios of the daily effective bid-ask spread over the event windows. There is clear evidence that spreads have dramatically increased after the announcement of the ICOs rules. During the event day, the mean of the spread ratio is 1.305 , with a highly significant $t$-statistic of 3.33 . For interval $[-1,+1]$, the mean ratio is 1.197 , which is significantly different from zero. We report that during the 3-day period of bad news, spreads are significantly higher than the pre-announcement period. However, from the event window $[-4,+4]$, the impact on spreads starts to diminish in magnitude. This is indicated by the value of 0.999 for the effective spread.

The illiquidity ratios, RtoV and RtoTR have clearly risen over the event window. In the $[-1,+1]$ interval the RtoV and RtoTR ratios are 1.238 and 1.299 respectively, with both ratios being highly significant. In the $[-3,+3]$ interval, we have mixed results with $R$ to $V$ being insignificant (t-statistic of 1.30) and RtoTR displaying significance at the 5\% level with a numerical value of 1.175 , and a corresponding t-statistic of 2.59 . In the 20 days after the announcement the negative effect is diminishing as all values are less than 1, regardless of the liquidity measurement used. Our results suggest that the negative news decreases the liquidity level of 
the cryptocurrency market, but this does not persist in the long run. With respect to trading volume, the ratio is 0.814 for the event day and 0.823 for the $[-1,+1]$ interval. This implies that the trading volume dramatically decreases around the announcement day. In Panel B we repeat the same test on the five largest cryptocurrencies. The results are quantitatively similar with Panel A, suggesting that investment in Bitcoin, Ethereum, Ripple, Bitcoin Cash and EOS has significantly declined as a result of the event.

\section{[INSERT TABLE 2 HERE]}

\section{Conclusion}

We analyze liquidity changes around the announcement of the Chinese government preventing ICOs in the cryptocurrency market on the $4^{\text {th }}$ of September 2017 . Using a sample of the 100 largest cryptocurrencies by market capitalization, we find evidence of significant but temporary liquidity changes in cryptocurrency markets. The abnormal return around the news announcement is negative and is associated with a return reversal in the long run. All measures show liquidity level decreases after the event. However, the liquidity impact is overturned in the long run. The results remain intact when we analyse the five largest cryptocurrencies (Bitcoin, Ethereum, Ripple, Bitcoin Cash and EOS). 


\section{References}

Amihud, Y., (2002), "Illiquidity and stock returns: Cross-section and time-series effects", Journal of Financial Markets, Vol.5, pp, 31-56.

Brauneis, A., and Mestel, R., (2018), "Price discovery of cryptocurrencies: Bitcoin and beyond", Economics Letters, Vol.165, pp.58-61.

Ciaian, P., Rajcaniova, M., and Kancs, D., (2016), "The economics of Bitcoin price formation", Applied Economics, Vol.48(19), pp. 1799-1815.

Corbet, S., Meegan, A., Larkin, C., Lucey, B., and Yarovaya, L., (2018), "Exploring the dynamic relationships between cryptocurrencies and other financial assets", Economics Letters, Vol. 165, pp.28-34.

Dwyer. G.P., (2015), "The economics of Bitcoin and similar private digital currencies", Journal of Financial Stability, Vol.17, pp.81-91.

Florackis,C., Gregoriou,A., and Kostakis,A., (2011)., "Trading frequency and asset pricing on the London Stock Exchange: Evidence firm a new price impact ratio", Journal of Banking \& Finance, Vol.35(12), pp.3335-3350.

Kristoufek, L., (2018), “On Bitcoin markets (in)efficiency and its evolution”, Physica A, Vol. 503, pp. 257-262.

Nakamoto,S., (2008), "Bitcoin: A peer-to-peer electronic cash system”, www. Bitccoi.org.

Zargar, F.N., and Kumar, D., (2019), "Informational inefficiency of Bitcoin: A study based on high-frequency data”, Research in International Business and Finance, Vol. 47, pp.344-353.

Zhang, S., Zhou, X., Pan, H., and Jia, J., (2019), "Cryptocurrency, confirmatory bias and news readability-evidence from the largest Chinese cryptocurrency exchange", Accounting and Finance, Vol.58, pp.1445-1468. 
Table 1. Price effects associated with the Chinese government preventing ICOs in the cryptocurrency market.

Table 1 provides the financial return changes from the Chinese government banning ICOs in the cryptocurrency market on the $4^{\text {th }}$ of September 2017. T-statistics are presented to show if cryptocurrency abnormal returns are significantly different from zero. Two tailed tests of significance are reported as follows, ${ }^{* * *}$ significance at $1 \%$, ${ }^{* *}$ significance at $5 \%$ and ${ }^{*}$ significance at the $10 \%$ level.

Panel A: 100 largest cryptocurrencies

Panel B: 5 largest cryptocurrencies (Bitcoin,

\begin{tabular}{l|llll}
\multicolumn{2}{|c}{} & & \multicolumn{3}{c}{ Ethereum, Ripple, Bitcoin Cash and } \\
\hline Event day & Abnormal returns (\%) & T-stat & Abnormal returns (\%) & T-stat \\
$\mathbf{- 5}$ & 0.439 & $6.49^{* * *}$ & 0.315 & $5.00^{* * * *}$ \\
$\mathbf{- 4}$ & 0.373 & $5.38^{* * *}$ & 0.247 & $4.63^{* * * *}$ \\
$\mathbf{- 3}$ & 0.402 & $5.96^{* * *}$ & 0.334 & $5.21^{\text {****}}$ \\
$\mathbf{- 2}$ & -0.013 & $-2.00^{*}$ & 0.258 & $4.82^{* * *}$ \\
$\mathbf{- 1}$ & -0.006 & $-1.88^{*}$ & -0.169 & $-2.87^{* *}$ \\
$\mathbf{0}$ & -4.265 & $-11.28^{* * *}$ & -5.330 & $-14.09^{* * * *}$ \\
$\mathbf{1}$ & -3.057 & $-9.99^{* * *}$ & -3.735 & $-11.04^{* * * *}$ \\
$\mathbf{2}$ & -3.533 & $-10.38^{* * *}$ & -3.048 & $-9.88^{* * *}$ \\
$\mathbf{3}$ & -0.266 & $-3.44^{* * *}$ & -1.266 & $-7.43^{* * *}$ \\
$\mathbf{4}$ & -0.389 & $-3.53^{* * *}$ & -0.565 & $-6.96^{* * *}$ \\
$\mathbf{5}$ & 0.003 & 1.26 & -0.000 & -1.00 \\
$\mathbf{( - 1 , + 1 )}$ & -7.327 & $-15.31^{* * *}$ & -9.234 & $-16.83^{* * * *}$ \\
$\mathbf{( - 2 , + 2 )}$ & -10.874 & $-17.84^{* * *}$ & -12.540 & $18.94^{* * *}$ \\
$\mathbf{( - 3 , + 3 )}$ & -11.739 & $-18.03^{* * *}$ & -13.472 & $19.73^{* * * *}$ \\
$\mathbf{( - 4 , + 4 )}$ & -11.755 & $-18.26^{* * *}$ & -13.790 & $20.44^{* * *}$ \\
$\mathbf{( - 5 , + 5 )}$ & -11.321 & $-17.20^{* * *}$ & -13.475 & $19.68^{* * *}$ \\
$\mathbf{1 0}$ & 0.458 & $5.29^{* * *}$ & 0.236 & $4.22^{* * *}$ \\
$\mathbf{2 0}$ & 0.639 & $6.13^{* * *}$ & 0.449 & $6.13^{* * *}$
\end{tabular}

Table 2. Liquidity effects associated with the Chinese government preventing ICOs in the cryptocurrency market.

Table 2 provides the liquidity changes from the Chinese government banning ICOs in the cryptocurrency market on the $4^{\text {th }}$ of September 2017. Liquidity is measured by the effective spread, the RtoV and RtoTR ratios and the trading volume. The null hypothesis is that the mean of the reported liquidity measure is equal to unity. This is examined using a standard t-test. ${ }^{* * *}$ significance at $1 \%,{ }^{* *}$ significance at $5 \%$ and ${ }^{*}$ significance at the $10 \%$ level.

\begin{tabular}{|c|c|c|c|c|c|c|c|c|}
\hline \multicolumn{9}{|c|}{ Panel A: 100 Cryptocurrencies } \\
\hline Event Day & $\begin{array}{l}\text { Effective } \\
\text { Spread }\end{array}$ & T-test & RtoV & T-test & RtoTR & T-test & $\begin{array}{l}\text { Trading } \\
\text { volume }\end{array}$ & T-stat \\
\hline $\mathbf{0}$ & 1.305 & $3.33^{* * *}$ & 1.293 & $2.87^{* *}$ & 1.360 & $3.48^{* * *}$ & 0.814 & $-4.16^{* * *}$ \\
\hline$(-1,+1)$ & 1.197 & $2.12^{* *}$ & 1.238 & $3.41^{* * *}$ & 1.299 & $3.87^{* * *}$ & 0.823 & $-3.89^{* * *}$ \\
\hline$(-2,+2)$ & 1.089 & $1.91^{*}$ & 1.264 & $2.93^{* *}$ & 1.030 & 1.56 & 0.916 & $-3.12^{* *}$ \\
\hline$(-3,+3)$ & 1.134 & $2.17^{*}$ & 1.003 & 1.30 & 1.175 & $2.59^{* *}$ & 0.895 & $-3.57^{* * *}$ \\
\hline$(-4,+4)$ & 0.999 & -1.27 & 0.977 & $-2.04^{*}$ & 0.984 & $-1.63^{*}$ & 0.926 & $-2.98^{* *}$ \\
\hline$(-5,+5)$ & 0.973 & $-1.65^{*}$ & 0.991 & -1.35 & 0.957 & $-2.08^{*}$ & 0.919 & $-3.00^{* *}$ \\
\hline$(0,+10)$ & 0.988 & $-1.73^{*}$ & 1.017 & $1.74^{*}$ & 0.939 & $-2.71^{* *}$ & 0.934 & $-2.18^{* *}$ \\
\hline$(0,+20)$ & 0.872 & $-3.49^{* * *}$ & 0.979 & $-2.32^{* *}$ & 0.948 & $-2.14^{*}$ & 0.941 & $-1.96^{*}$ \\
\hline \multicolumn{9}{|c|}{ Panel B: 5 Largest Cryptocurrencies (Bitcoin, Ethereum, Ripple, Bitcoin Cash and EOS) } \\
\hline 0 & 1.316 & $3.65^{* * *}$ & 1.300 & $3.54^{* * *}$ & 1.353 & $3.99^{* * *}$ & 0.802 & $-4.67^{* * *}$ \\
\hline$(-1,+1)$ & 1.201 & $2.30^{* *}$ & 1.276 & $2.93^{* *}$ & 1.301 & $3.54^{* * *}$ & 0.819 & $-4.02^{* * *}$ \\
\hline$(-2,+2)$ & 1.114 & $1.95^{*}$ & 1.269 & $2.91^{* *}$ & 1.096 & 1.23 & 0.894 & $-3.18^{* *}$ \\
\hline$(-3,+3)$ & 1.055 & $1.63^{*}$ & 1.164 & $2.43^{* *}$ & 0.999 & $-1.58^{*}$ & 0.900 & $-2.90^{* *}$ \\
\hline$(-4,+4)$ & 1.003 & $1.42^{*}$ & 1.001 & 1.29 & 1.001 & 1.14 & 0.884 & $-3.41^{* * *}$ \\
\hline$(-5,+5)$ & 0.986 & $-1.50^{*}$ & 0.984 & -1.23 & 0.990 & $-1.43^{*}$ & 0.931 & $-2.56^{* *}$ \\
\hline$(0,+10)$ & 0.990 & $-1.62^{*}$ & 0.913 & -0.96 & 0.913 & -1.26 & 0.924 & $-2.77^{* *}$ \\
\hline$(0,+20)$ & 0.983 & $-1.46^{*}$ & 0.934 & -1.11 & 0.961 & 1.35 & 0.969 & $-1.93^{*}$ \\
\hline
\end{tabular}

